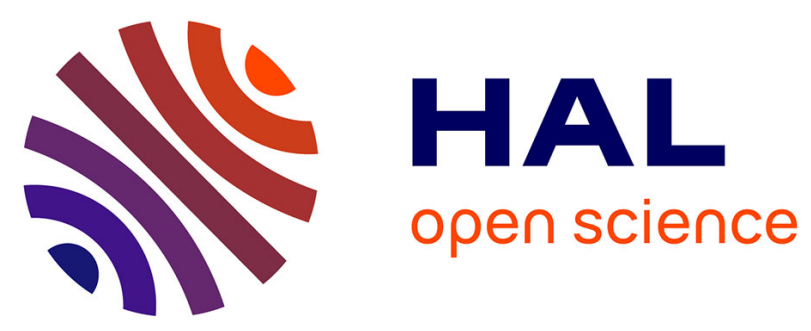

\title{
Two domains of MalT, the activator of the Escherichia coli maltose regulon, bear determinants essential for anti-activation by MalK
}

\author{
Evelyne Richet, Nicolas Joly, Olivier Danot
}

\section{- To cite this version:}

Evelyne Richet, Nicolas Joly, Olivier Danot. Two domains of MalT, the activator of the Escherichia coli maltose regulon, bear determinants essential for anti-activation by MalK. Journal of Molecular Biology, 2005, 347 (1), pp.1-10. 10.1016/j.jmb.2005.01.011 . pasteur-03266382

\section{HAL Id: pasteur-03266382}

\section{https://hal-pasteur.archives-ouvertes.fr/pasteur-03266382}

Submitted on 21 Jun 2021

HAL is a multi-disciplinary open access archive for the deposit and dissemination of scientific research documents, whether they are published or not. The documents may come from teaching and research institutions in France or abroad, or from public or private research centers.
L'archive ouverte pluridisciplinaire HAL, est destinée au dépôt et à la diffusion de documents scientifiques de niveau recherche, publiés ou non, émanant des établissements d'enseignement et de recherche français ou étrangers, des laboratoires publics ou privés.

\section{(1) $(1) \Theta$}

Distributed under a Creative Commons Attribution - NonCommercial - NoDerivatives 44.0 


\section{Two domains of MalT, the activator of the Escherichia coli maltose regulon, bear determinants essential for anti-activation by MalK}

Evelyne Richet *, Nicolas Joly and Olivier Danot

Unité de Génétique Moléculaire, URA CNRS 2172

Institut Pasteur,

25, rue de Dr. Roux,

75724 Paris Cedex 15

France

*Corresponding author

Tel: 33140613680

Fax: 33145688960

E-mail: erichet@pasteur.fr

Keywords: MalK / MalT / transcription activator / ABC transporter / maltose regulon Running title: malT mutations preventing inhibition by MalK 


\section{SUMMARY}

MalT, the dedicated transcriptional activator of the maltose regulon in Escherichia coli, is subject to multiple controls. Maltotriose, the inducer, promotes MalT self-association, a critical step in promoter binding, whereas three proteins acting as negative allosteric effectors (MalK, the ABC-component of the maltodextrin transporter, MalY, and Aes) antagonize maltotriose binding. All of these regulatory signals are integrated by a novel signal transduction module that comprises three out of the four MalT structural domains: DT1, the ATP-binding domain which contains determinants recognized by the negative effectors, DT2, and DT3, the maltotriose binding domain. For a better insight into the role of DT3 in signal integration, we PCR mutagenized the DT3-encoding region and screened for gain of function mutations in a malK ${ }^{+}$strain in the absence of repression by MalY or Aes. Most of the mutations isolated alter one of seven residues that are located in DT3 helices 10 and 11 or in the turn between them and delineate a surface-exposed motif. In vivo and in vitro analyses revealed that the substitutions altering the so-called H10/H11 motif do not affect the ability of MalT to activate transcription or its sensitivity to MalY and Aes, but dramatically decrease its sensitivity to MalK. We propose that MalT/MalK interaction might involve two distinct contact sites on each partner. These sites would be located in DT1 and DT3 of MalT, and in the nucleotide binding domain and the regulatory domain of MalK. Such a two-point interaction model would explain how the regulatory activity of MalK might be coupled to transport. 


\section{INTRODUCTION}

MalT, the dedicated transcriptional activator of the maltose regulon in Escherichia coli, is the prototype of a family of $\sim 100 \mathrm{kDa}$ transcriptional activators that belong to the STAND (Signal Transduction ATPases with Numerous Domains) class of P-loop ATPases ${ }^{1}$. Transcription activation by MalT involves MalT self-association ${ }^{2}$, cooperative binding to copies of the MalT recognition motif that are present in target promoters ${ }^{3,4,5}$, and stimulation of open complex formation by RNA polymerase ${ }^{6}$. One distinctive feature of MalT is the multiplicity of regulatory signals that modulate MalT self-association: MalT multimerization is stimulated by maltotriose, the inducer, ${ }^{7}$ and inhibited by three proteins, Aes, MalK and MalY, which act as negative allosteric effectors ${ }^{8,9,10}$. Control of MalT activity by MalK is thought to couple induction of the maltose regulon to the entry of maltodextrins into the cell ${ }^{11}$. The physiological roles of the controls exerted by Aes, an acyl esterase ${ }^{12}$ and MalY, a $\beta \mathrm{C}-\mathrm{S}$ lyase ${ }^{13}$, are presently unknown. According to the current model, MalT exists in an equilibrium between an inactive monomeric form and an active monomeric form that is prone to multimerization. The inactive form is stabilized by MalK, MalY or Aes, whereas the active form is stabilized by maltotriose which thus competes with the negative effectors for binding to MalT 2, 8, 9, 10 . MalT also binds and hydrolyses ATP into ADP, with ATP and ADP differentially affecting the interplay between maltotriose and the negative effectors. Efficient MalT multimerization requires ATP or ADP in addition to maltotriose, with ATP being more effective than ADP in driving MalT self-association in the presence of maltotriose, indicating that the maltotriose/ATP-bound form is the transcriptionally active species ${ }^{2}$. Negative effectors also bind MalT in the presence of ATP or ADP. However, maltotriose does not relieve MalT inhibition by Aes or MalY in vitro in the presence of ADP, whereas it can do so in the presence of ATP ${ }^{8,9}$. These data suggest that the ATPase activity of MalT might play a role in the competition between the positive and negative effectors.

Biochemical and structural studies have provided insight into the structure of MalT. The protein comprises four domains: DT1 (residues 1-241), DT2 (residues 250-436), DT3 (residues 437-806) and DT4 (residues 807-901) ${ }^{14,15}$. Integration of the regulatory signals is ensured by the three N-terminal domains, DT1, DT2 and DT3, which constitute a novel signal transduction module that is the signature of the MalT subfamily of STAND ATPases ${ }^{1,15}$. DT1 binds and hydrolyses ATP ${ }^{15}$. It also contains surface determinants involved in the binding of Aes, MalK and MalY ${ }^{9,}{ }^{10,16}$. DT3 binds maltotriose ${ }^{15}$. According to the X-ray structure of DT3 ${ }^{17}$, the main DT3 subdomain corresponds to a new fold that comprises eight copies of a two-helix bundle motif arranged in a right-handed superhelix and delimiting a tunnel large enough to form a potential maltotriose binding site. The super-helix is followed by a three-helix subdomain. DT4, a member of the LuxR-type DNA-binding domain family, contains the DNA binding site and presumably recruits RNA polymerase ${ }^{6}$. The 
DT1/DT2/DT3 module is thought to respond to incoming regulatory signals via a change of its quaternary structure that alters the ability of DT4, the output domain, to bind cooperatively to the array of MalT sites present in the target promoters ${ }^{15}$. Cryo-E.M. analyses showed that, in the active form, i.e. in the presence of both ATP and maltotriose, MalT monomers consist of a globular domain connected by a peduncle to an inner domain, that mediates self-association into polydisperse homopolymers ${ }^{18}$.

For a better insight into the role played by DT3 in signal integration or response, we searched for gain of function mutations affecting DT3. We anticipated that such an appproach might allow the identification of amino acids playing a key role in conformational changes, in effector binding, in intra-molecular interactions, or in inter-molecular interactions. The search, which was performed under conditions in which MalT activity was repressed by MalK, led to the identification of a surface region on DT3 that is specifically involved in the control of MalT activity by MalK. 


\section{RESULTS}

\section{Search for gain of function mutations affecting DT3}

The DNA region of malT encoding DT3 and the first twenty residues of DT4 (DT3-DT4') was mutagenized by error prone PCR ${ }^{19}$ and cloned in a full length malT in a single copy plasmid (pOM180). Up mutations in DT3 were searched for in a $\Delta$ malT malK reporter strain (pop7191), the chromosome of which contains the lac operon under the control of malEp $\triangle 92$, a MalT-dependent promoter. When MalT is provided in trans by pOM180, malEp $\triangle 92$ activity is low in the absence of external maltodextrins as a result of MalT repression by MalK ${ }^{\mathrm{a}}$. Hence, pop7191 (pOM180) produces pink colonies on MacConkey lactose agar. Because the activity of malEp $\Delta 92$ is limited by the concentration of active MalT forms under these conditions, one can identify gain of function mutations in malT by looking for red colonies on MacConkey lactose plates. Indeed, red clones $\left(\mathrm{Lac}^{+}\right)$appeared when pop7191 was transformed with the PCR mutagenized pOM180 plasmid bank and plated on MacConkey lactose plates. Subcloning of the DT3-DT4' region from the mutant candidate plasmids in pOM181, a variant of pOM180, showed that the up phenotype was associated with the mutagenized region of the plasmid in $\sim 90 \%$ of the cases. Sequencing of the mutagenized regions of 27 pOM181 up mutants led to the identification of 19 different mutants, including six double mutants (Table 1). Remarkably, most of the mutants contained an amino acid substitution altering one of seven clustered residues (R634, E636, N637, L638, L639, and R656) that are located in DT3 helices 10 and 11 (i.e. the second helix of the fifth bundle and the first helix of the sixth bundle), or in the turn between them (G640) (Figure 1). These residues are surface exposed or are located close to the surface of DT3, which suggests that these substitutions alter a unique surface determinant, hereafter called the H10/H11 motif. Except for one case (G640R), the double mutants all contained a substitution found independently in a single mutant, strongly suggesting that the second substitution is silent with regards the observed phenotype. Therefore, further analysis was restricted to the single mutants altered in the DT3 H10/H11 area. G640R was not further characterized because of the existence of a single mutant that exhibited a different substitution at the same position (G640E). The three up mutants (M517K/H808R, Q565R, and F810L) with substitutions unrelated to $\mathrm{H} 10$ and $\mathrm{H} 11$ will be the subject of an independent study.

\section{All of the amino acid substitutions altering the DT3 H10/HT11 area specifically affect MalT repression by MalK}

To quantify the effect of the DT3 up mutations on the activity of MalT, we measured the amount of $ß$-galactosidase synthesized by two isogenic reporter strains when transformed

\footnotetext{
${ }^{\text {a }}$ Although strain pop7191 carries the malY and aes genes, they are phenotypically MalY ${ }^{-}$and Aes ${ }^{-}$because these genes are not expressed under these conditions ${ }^{12,13}$.
} 
with pOM181 or variants thereof and grown in minimal medium supplemented with glycerol: the $\Delta$ malT malK $^{+}$strain used above (pop7191), in which MalT was repressed by MalK, and an isogenic $\Delta$ malT $\Delta$ malK strain (pop7190), in which the activity of MalT was unrepressed. As illustrated in Figure 2A, the DT3 up mutations had little effect on MalT activity in the unrepressed context; in the $\Delta$ malT $\Delta$ malK reporter strain, the activity levels obtained with the mutants were within $25 \%$ of the activity level observed with pOM181. In contrast, these mutations caused a 5 to 14 times increase in the activity of malEp $\triangle 92$ in the presence of MalK, which resulted in a dramatic decrease in the repression by MalK from 12.5 for w.t. MalT to 1.0-1.9 for the mutants. Altogether, these data suggested that the up mutations altering the DT3 H10/H11 region interfere, partially or fully, with MalT inhibition by MalK.

We then asked whether the up mutations also relieve MalT inhibition by MalY and by Aes, two other negative effectors of MalT. The effect of the DT3 up mutations on MalT repression by MalY was assessed by measuring the levels of $\beta$-galactosidase made by an isogenic $\Delta$ malT $\Delta$ malK malI::Tn10 strain (pop7192) carrying pOM181 or derivatives thereof. The inactivation of mall, which encodes the repressor of the malX malY operon, causes constitutive expression of the malX malY operon ${ }^{13}$ and a mall::Tn10 strain is therefore phenotypically MalY ${ }^{+}$. The level of activity observed with w.t. MalT in the presence of MalY was $17 \%$ of the unrepressed level under these conditions (Figure $2 \mathrm{~A}$ ). Interestingly, the up mutations had little effect on the activity of malEp $\Delta 92$ in the presence of MalY (Figure 2A). The $\beta$-galactosidase levels ranged from 0.7 to 1.4 times that obtained with wild-type MalT, which contrasts with the 5 to 14 times stimulatory effect observed in the presence of MalK.

The effect of the up mutations on MalT repression by Aes was monitored by measuring the levels of $\beta$-galactosidase made by the $\Delta$ malT $\Delta$ malK strain (pop7190) carrying both pOM181 (or derivatives thereof) and a second plasmid, pACS2 which encodes Aes, or the empty vector plasmid (pACYC184). Four representative up mutations were tested: R634S, E636G, N637T, and L639P. The level of $\beta$-galactosidase observed with wild-type MalT when repressed by Aes corresponded to $4 \%$ of the unrepressed level (Figure 2B). Again, in contrast to what observed with MalK, the up mutations tested had little effect on malEp $\Delta 92$ activity in the presence of Aes (Figure 2B).

In conclusion, all of the substitutions altering residues R634, E636, N637, L638, L639, G640 and R656 enhance MalT activity under conditions of repression by MalK but have no significant effect on MalT activity in the absence of repressor or in the presence of MalY or, in the cases of residues 634, 636, 637 and 639, in the presence of Aes. These observations confirm that these mutations modify a single determinant, and strongly suggest that this determinant plays a key role in MalT inhibition by MalK. 


\section{The malT-N637T mutation interferes with MalT inhibition by MalK in vitro}

To confirm this interpretation, we determined whether N637T, one of the surface exposed substitutions altering the H10/H11 surface determinant (see Discussion), also relieves MalT repression by MalK in vitro. MalT inhibition by MalK was assessed by monitoring its ability to activate open complex formation in the presence of MalK at $m a l P p$, a promoter whose activity strictly depends on MalT. The amount of open complexes formed was quantified by using an abortive initiation assay. The assays were performed in the presence of a limiting concentration of MalT to ensure that inhibition by MalK, if any, would be detected $\left(\right.$ see $^{10}$ ). As shown in Figure 3, activation of open complex formation by MalT-N637T is not depressed by MalK at a concentration that reduced activation by wild-type MalT by $70 \%$ (Figure 3). In addition, similar in vitro repression assays performed with Aes and MalY confirmed that the MalT-N637T substitution does not alter MalT response to the other two negative effectors (Figure 4).

Since MalK is known to bind DT1, another domain of MalT ${ }^{10}$, it was possible that the H10-H11 motif is important for MalK function (i.e. repression of MalT activity) but not for its binding to MalT. Thus, we examined the ability of the MalT-N637T variant to interact with MalK by performing affinity chromatography experiments. Purified MalT protein was chromatographed on a Ni-NTA column preloaded with His-tagged MalK, and after extensive washing, MalK was eluted with imidazole. As shown in Figure 5, a fraction of the wild-type MalT protein was retained on the column and co-eluted with MalK. The retention observed is significant because it completely disappears when affinity chromatography is performed with a MalK variant (G346S) defective in MalT repression ${ }^{10}$. Interestingly, the N637T variant was not retained on the column, thereby demonstrating that the N637T substitution, directly or indirectly, impedes the interaction with MalK. 


\section{DISCUSSION}

The search for gain of function mutations in DT3 under conditions of MalT inhibition by MalK revealed a surface-exposed determinant that is specifically involved in MalT control by MalK. The up mutations alter either of seven clustered residues in helices 10 and 11 or in the turn between them ${ }^{17}$. These two helices belong to adjacent two-helix bundles that are part of the wall of the DT3 superhelix. In vivo and in vitro characterization of the mutated MalT proteins provides compelling evidence that the substitutions do not significantly impair the intrinsic ability of MalT to activate transcription initiation, but specifically render MalT resistant to the inhibition by MalK. Indeed, in vivo, the substitutions scarcely affect MalT activity in a no-repression context as well as MalT response to MalY or, in the four cases tested, to Aes. In contrast, all of the substitutions partially or entirely alleviate MalT inhibition by MalK. Furthermore, the N637T substitution, which alters a surface-exposed residue, does not affect MalT activity in vitro, or its sensitivity to the inhibition by MalY or Aes, but fully prevents MalT inhibition by MalK.

The substituted amino-acids are either part of a surface determinant - the H10/H11 motif - playing a crucial role in MalT repression by MalK or contribute to the correct exposition of this determinant ${ }^{17}$. Indeed, two residues in helix 10, R634 and N637, are decidly surface-exposed. Residues L638 and L639 at the end of helix 10 are not surfaceexposed but most likely contribute to the proper secondary structure and orientation of helix 10. Their replacement by a proline is likely to cause a change in the local structure. Similarly, the substitution of G640 by a glutamate or an arginine might alter the structure of the turn or inactivate the H10/H11 motif by the introduction of a charge and/or a bulkier residue. The glutamate at position 636 and the arginine at position 656 probably contribute to the packing of the fifth and sixth two-helix bundles since they form a salt bridge. Residue D636, which is not completely buried, might also be part of the H10/H11 surface motif. Hence, its substitution with a glycine might affect MalT repression by MalK because it disrupts the salt bridge between helices 10 and 11 and/or because it alters the H10/H11 motif surface. As indicated by the absence of an effect on the intrinsic ability of MalT to activate transcription initiation, the amino acid changes caused by the up mutations do not grossly disturb DT3 structure nor alter a DT3 surface element that would play a key role in the stabilization of the active form of MalT or any subsequent step.

What is the function of the H10/H11 surface motif in MalT inhibition by MalK? Based on the observation that the N637T substitution prevents MalK from interacting with MalT in vitro, we infer that the H10/H11 surface element is involved in MalK binding. DT1 is known to contain a determinant that is specifically bound by the MalT-binding site in the C-terminal, regulatory domain of MalK ${ }^{10,20}$. The interaction observed in vitro between MalK and the isolated DT1 domain is indeed disrupted by malK-G346S, one of the mutations defining the 
MalT-binding site. Thus, the H10/H11 motif defined here might represent an additional determinant recognized by the $\mathrm{ABC}$ component of the maltodextrin transporter. The MalK binding site might result from the juxtaposition of the DT1 and DT3 determinants that would occur when MalT is in the inactive form. Alternatively, the DT1 and DT3 determinants recognized by MalK might not be close to each other in the inactive form of MalT and might correspond to two distinct patches recognized by MalK. If the latter hypothesis is correct, the MalK protein must have another patch involved in MalT recognition, in addition to that already identified in its regulatory domain ${ }^{20}$. Such a two-point interaction model is actually supported by the effect of the P72L substitution in the nucleotide binding domain of MalK, that specifically confers a regulation-minus phenotype ${ }^{20}$. Residue P72 is located in a large loop that is surface-exposed in isolated $\mathrm{MalK}^{21}$ and that should be at least partially accessible when MalK is part of the transporter complex, if the overall topology of $\mathrm{MalFGK}_{2}$ resembles that of BtuCD, the only bacterial ABC-importer with a known three dimensional structure ${ }^{22}$. This residue might therefore contribute to the structure of a determinant located in the MalK nucleotide binding domain and recognized by the H10/H11 motif of MalT. Furthermore, the existence of two distinct patches on MalK, in the regulatory domain and in the nucleotide binding domain, respectively, would help explain how the ability of MalK to interact with MalT might be controlled by the activity of the transporter, as proposed by Panagiotidis et $a l .{ }^{23}$. Indeed, the hypothised coupling is not supported by the X-ray structural data of Chen et al. ${ }^{21}$ if one assumes that formation of a MalT/MalK complex is only mediated by the MalT binding site identified in the MalK regulatory domain. The crystal structures of free and ATPbound MalK dimers suggest that ATP hydrolysis is not accompanied by conformational changes of the C-terminal domain of MalK. In contrast, the existence of two distant half-sites on MalK whose spatial relationship depends on whether MalK is in the open or the closed conformation, i. e., on whether the transporter is resting or actively transporting maltodextrins ${ }^{24}$, explains how the ability of MalK to interact with MalT might be coupled to the absence of transport.

Affinity chromatography experiments revealed that MalK does not interact with DT3 when isolated (N. J., unpublished), which argues against the hypothesis that the H10/H11 surface motif is recognized by MalK. However, the conformation of the isolated DT3 domain might differ from that of DT3 in the full length MalT context. This could explain why isolated DT3 does not interact with MalK. Alternatively, the interaction between MalK and DT3 might be too weak to be detected by affinity chromatography. In this respect, it might be worth noting that the DT1/MalK interaction is weak ${ }^{10}$.

Finally, it remains possible that the H10/H11 motif is not recognized by MalK but, instead, is involved in the stabilization of the MalT inactive form recognized by MalK. However, such a hypothesis implies that the active form of MalT would be in equilibrium with at least two different inactive forms of MalT and that only the form recognized by MalK 
would be stabilized by an intramolecular domain/domain interaction mediated by the DT3 H10/H11 motif.

The superhelix fold, SUPR, that constitutes most of DT3 is related to the tetratricopeptide repeat (TPR) fold ${ }^{17}$, a structure that often mediates protein-protein interactions ${ }^{25}$. This work shows that this property can be extended to the SUPR fold. Interestingly, whereas most TPR proteins interact with their binding partner through the interior surface of the superhelix, in this case the interaction patch (either with MalK or with a domain of MalT) is located on the exterior surface of DT3. This shows the versatility of this kind of fold in terms of protein-protein interaction. Furthermore, since the interior of the tunnel is thought to bind maltotriose (O. D., unpublished results), our finding might provide a simple explanation for the competition between MalK and maltotriose for MalT binding. Indeed, it is easy to imagine how interaction of MalK or another domain of MalT with the exterior cleft separating the fifth and sixth helix bundles could change the curvature of the inner surface of the tunnel and preclude the binding of maltotriose, if it does bind in the tunnel, and, reciprocally, how maltotriose could prevent the H10/H11 motif from binding its target. Binding of two ligands on both sides of a wall made of two alpha-helix bundles is an elegant and simple way to generate allostery, and one could expect to find other examples of it. 


\section{MATERIALS AND METHODS}

Strains. All of the strains used (Table 1) are derivatives of the E. coli K-12 MC4100 strain $^{26}$.

Plasmids. pACS2 is a derivative of pACYC184 that contains the aes gene under the control of its own promoter ${ }^{16}$. pOM180 was derived from pOM24, which contains the EcoRI-EcoRI malT fragment from pOM26 ${ }^{27}$ cloned in the EcoRI site of pJM241 (a single-copy R1 run-away plasmid) ${ }^{28}$, by the introduction of a silent KpnI site right after the $20^{\text {th }}$ codon of DT4 (an A $->$ G change at position 2478 in the malT coding sequence). pOM181 is a derivative of pOM180 in which the 21 bp BamHI-BamHI fragment, which contains two SmaI sites and an EcoRI site, was deleted. Plasmid pOM204 was constructed by inserting the PstI-EcoRI malT fragment of pOM180 between the PstI and EcoRI sites of a pBR322 variant with a silent A -> G change (obtained by inverse PCR mutagenesis) at position 147 in the tet gene that inactivates the unique NheI site. Plasmid pOM2 malTp 7 is a pBR322 derivative containing the malT gene under the control a malTp promoter variant bearing an up mutation ${ }^{4}$. pOM7 malTp 7 malT-N637T was constructed by replacing the HpaIEcoRI fragment of pOM2 malTp 7 by the HpaI-EcoRI fragment of pOM181 malT-N637T. In all of the constructs but the pBR322 variant, the sequences of the regions resulting from PCR amplification were verified. Antibiotic concentrations were $5 \mu \mathrm{g} / \mu \mathrm{l}$ of tetracyclin, $30 \mu \mathrm{g} / \mu \mathrm{l}$ of ampicillin, and $30 \mu \mathrm{g} / \mathrm{ml}$ of chloramphenicol.

PCR mutagenesis. The reaction mixture $(50 \mu \mathrm{l})$ contained $10 \mathrm{mM}$ Tris- $\mathrm{HCl}(\mathrm{pH} 8.5)$, $50 \mathrm{mM} \mathrm{KCl}, 1.5 \mathrm{mM} \mathrm{MgCl} 2,50 \mu \mathrm{M}$ each dNTP, $0.14 \mu \mathrm{g}$ ClaI-linearized pOM204 DNA, $2 \mu \mathrm{M}$ primer MT56 (5' - AAATCCGCAGTTGGTGTTA - 3'), $2 \mu \mathrm{M}$ primer MT57 (5' GCCCCAGTACCTGCCA - 3'), and $2.5 \mathrm{u}$ Ampli Taq DNA polymerase (Applied Biosystems). After a four-cycle amplification, DNA was extracted, digested with NheI and KpnI, and ligated with the purified $\sim 8.8$-kb KpnI-NheI pOM180 fragment. Cells pop7191 were electroporated with the ligation products, plated on McConkey lactose + ampicillin agar and incubated at $30^{\circ} \mathrm{C}$ for $24 \mathrm{~h}$. Red clones were picked, reisolated on the same medium, and plasmid DNA was extracted from the candidates. The NheI-KpnI fragment was then subcloned between the corresponding sites of pOM181 to verify that the up phenotype was associated with the DT3 encoding fragment, and was sequenced.

$\boldsymbol{\beta}$-galactosidase assays. Strains pop7190, pop7191 or pop7192 harboring derivatives of pOM181 were grown in M9 medium ${ }^{29}$ supplemented with $0.4 \%$ glycerol, $0.01 \%$ tryptophane, $1 \mu \mathrm{g} / \mathrm{ml}$ thiamine, $0.5 \% \mathrm{~L}$ broth, and $30 \mu \mathrm{g} / \mathrm{ml}$ ampicillin. Strain pop7190 carrying pOM181 derivatives and pACYC184 (or pACS2) were cultivated in the same 
medium as above plus $30 \mu \mathrm{g} / \mathrm{ml}$ chloramphenicol The cultures were inoculated to a low cell density $\left(A_{600}=10^{-4}\right)$ and grown overnight at $30^{\circ} \mathrm{C}$ up to $A_{600}=0.6-0.9$. B-galactosidase was assayed at $28^{\circ} \mathrm{C}$ as described by Miller ${ }^{30}$, by using chloroform and $0.0037 \%$ SDS to disrupt the cells. The specific activity is given in Miller units (U). All values represent the average of assays performed in duplicate on at least two independent cultures, and are corrected for the background levels ( 6 to $8 \mathrm{U}$ ). The variations observed between independent experiments did not exceed $20 \%$.

Proteins. Wild-type MalT was purified in the presence of ATP from pop3657 (pOM2 malTp7), as described ${ }^{4}$. MalT-N637T was purified from pop7164 (pOM2 malTp7 malT-N637T) according to the same procedure with some modifications for the chromatography on propyl-agarose; the adsorption, washing, and elution steps were performed in the presence of $1,0.85$, and $0.35 \mathrm{M}\left(\mathrm{NH}_{4}\right)_{2} \mathrm{SO}_{4}$, respectively. ATP-free MalT was prepared by precipitating the purified protein with ammonium sulfate and filtering the resuspended material through a G-75 Sephadex column as described ${ }^{2}$. ATP-free MalT was used through out this work. MalT-N637T was about $80 \%$ as active as wild-type MalT, when activation of open complex formation at malPp was assayed (see hereafter). The MalY protein was obtained from C. Steegborn; its buffer was changed as described ${ }^{8}$. The Aes protein, which was His-tagged at its $\mathrm{N}$ terminus and prepared as described ${ }^{9}$, was obtained from A. Schlegel (University of Konstanz, Germany). The MalK protein, with a His-tag at its $\mathrm{N}$-terminus, was purified as described ${ }^{10}$. RNA polymerase holoenzyme $\left(E \sigma^{70}\right)$ was from Epicentre (Madison, USA).

Abortive initiation assay. MalT was preincubated at $30{ }^{\circ} \mathrm{C}$ for $15 \mathrm{~min}$ in $18 \mu \mathrm{l}$ of reaction mixture containing $40 \mathrm{mM}$ HEPES-KOH ( $\mathrm{pH}$ 8.0), $9 \mathrm{mM}$ Tris- $\mathrm{HCl}(\mathrm{pH} 7.7), 27 \mathrm{mM}$ tri-potassium citrate, $17 \mathrm{mM} \mathrm{NaCl}, 11 \mathrm{mM}$ magnesium acetate, $0.11 \mathrm{mM}$ EDTA, $1.1 \mathrm{mM}$ dithiothreitol, $22 \mathrm{mM}$ imidazole, $230 \mu \mathrm{g} \cdot \mathrm{ml}^{-1}$ acetylated bovine serum albumin (Sigma), $5 \mathrm{nM}$ malPp DNA fragment, $0.5 \mathrm{mM}$ AMP-PNP and, when indicated, Aes, MalK, or MalY. Two microliters of RNA polymerase $(0.13 \mu \mathrm{M}$ in $40 \mathrm{mM}$ HEPES-KOH $(\mathrm{pH} 8.0), 33 \mathrm{mM}$ tri-potassium citrate, $1 \mathrm{mM}$ dithiothreitol, and $0.1 \mathrm{mg} \cdot \mathrm{ml}^{-1}$ acetylated bovine serum albumin) was added, and the mixture was incubated for $15 \mathrm{~min}$ at $30^{\circ} \mathrm{C}$. The synthesis of abortive products (ApApC) was initiated by adding $2 \mu \mathrm{l}$ of a solution containing $5 \mathrm{mM}$ ApA, $0.5 \mathrm{mM}$ $\left[\alpha-{ }^{32} \mathrm{P}\right] \mathrm{CTP}\left(0.2 \mathrm{Ci} \cdot \mathrm{mmol}^{-1}\right)$ and $500 \mu \mathrm{g} \cdot \mathrm{ml}^{-1}$ heparin (H-0880; Sigma) and allowed to proceed for $15 \mathrm{~min}$ at $30{ }^{\circ} \mathrm{C}$ (heparin blocks open complex formation by trapping free RNA polymerase). The reaction products were separated from free $\left[\alpha-{ }^{32} \mathrm{P}\right] \mathrm{CTP}$ by chromatography on Whatman $3 \mathrm{MM}$ paper as described ${ }^{31}$. The chromatograms were dried and scanned on a PhosphorImager, and the amount of ApApC synthesized was quantified. The malPp DNA 
template is a $320 \mathrm{bp}$ fragment containing the malPp promoter (from 154 to +130 ), prepared as described ${ }^{8}$.

Affinity Chromatography with immobilized MalK. A soluble extract was prepared from a $10-\mathrm{ml}$ suspension of bacteria $\left(A_{600}=100\right)$ overproducing His-tagged MalK as described ${ }^{10}$. Affinity chromatography was performed at $4{ }^{\circ} \mathrm{C}$ in Micro Biospin ${ }^{\circledR}$ Bio-Rad columns packed with $50 \mu \mathrm{l}$ of Ni-NTA-agarose (Qiagen). Solutions were passed through the columns by spinning at $5 \mathrm{~g}$ for $30 \mathrm{sec}$ in a benchtop centrifuge. The columns were equilibrated with buffer A (20 mM Tris- $\mathrm{HCl}(\mathrm{pH} 7.7), 150 \mathrm{mM} \mathrm{NaCl}$ and $\left.5 \mathrm{mM} \mathrm{MgCl}_{2}\right)$ and loaded with $2 \mathrm{ml}$ of soluble extract. The columns were washed with buffer A ( 5 x $100 \mu \mathrm{l})$, and buffer $\mathrm{A}+40 \mathrm{mM}$ imidazole $(5 \times 100 \mu \mathrm{l})$. The MalK-loaded column was then equilibrated with buffer A $+2 \mathrm{mM} \mathrm{ATP}(2 \times 100 \mu \mathrm{l})$, and purified MalT (200 $\mu \mathrm{l}$ at $0.5 \mathrm{mg}^{-1} \mathrm{ml}^{-1}$ in buffer A $+2 \mathrm{mM} \mathrm{ATP}$ ) was allowed to flow through the column. Unbound proteins were washed out with $5 \times 100 \mu$ of buffer A $+40 \mathrm{mM}$ imidazole $+2 \mathrm{mM}$ ATP. His-tagged MalK was eluted with $4 \times 100 \mu \mathrm{l}$ of buffer $\mathrm{A}+500 \mathrm{mM}$ imidazole. 100- $\mu$ l fractions were collected, starting from the MalT washing step and analyzed by $12 \%$ SDS-PAGE (acrylamide:bisacrylamide, $37.5: 1)$. 


\section{Acknowledgements}

We thank the students of the 2002 Institut Pasteur General Microbiology Course for their contribution to this work. We are grateful to A.L. Davidson for suggesting the two-point MalT/MalK interaction model, and to A. Schlegel and C. Steegborn for providing Aes and MalY, respectively. We also thank T. Pugsley for his interest in this work and critical reading of the manuscript. N. J. was the recipient of a fellowship from the Ministère Délégué à la Recherche et aux Nouvelles Technologies. 


\section{REFERENCES}

1. Leipe, D. D., Koonin, E. V. \& Aravind, L. (2004). STAND, a Class of P-Loop NTPases Including Animal and Plant Regulators of Programmed Cell Death: Multiple, Complex Domain Architectures, Unusual Phyletic Patterns, and Evolution by Horizontal Gene Transfer. J. Mol. Biol. 343, 1-28.

2. Schreiber, V. \& Richet, E. (1999). Self-association of the Escherichia coli transcription activator MalT in the presence of maltotriose and ATP. J. Biol. Chem. 274, 33220-6.

3. Vidal-Ingigliardi, D., Richet, E. \& Raibaud, O. (1991). Two MalT binding sites in direct repeat. A structural motif involved in the activation of all the promoters of the maltose regulons in Escherichia coli and Klebsiella pneumoniae. J. Mol. Biol. 218, 323-334.

4. Danot, O. \& Raibaud, O. (1994). Multiple protein-DNA and protein-protein interactions are involved in transcriptional activation by MalT. Mol. Microbiol. 14, 335-346.

5. Richet, E. (1996). On the role of the multiple regulatory elements involved in the activation of the Escherichia coli malEp promoter. J. Mol. Biol. 264, 852-862.

6. Danot, O., Vidal-Ingigliardi, D. \& Raibaud, O. (1996). Two amino acid residues from the DNA-binding domain of MalT play a crucial role in transcriptional activation. $J$. Mol. Biol. 262, 1-11.

7. Raibaud, O. \& Richet, E. (1987). Maltotriose is the inducer of the maltose regulon of Escherichia coli. J. Bacteriol. 169, 3059-3061.

8. Schreiber, V., Steegborn, C., Clausen, T., Boos, W. \& Richet, E. (2000). A new mechanism for the control of a prokaryotic transcriptional regulator: antagonistic binding of positive and negative effectors. Mol. Microbiol. 35, 765-76.

9. Joly, N., Danot, O., Schlegel, A., Boos, W. \& Richet, E. (2002). The Aes protein directly controls the activity of MalT, the central transcriptional activator of the Escherichia coli maltose regulon. J. Biol. Chem. 277, 16606-13.

10. Joly, N., Böhm, A., Boos, W. \& Richet, E. (2004). MalK, the ATP-binding cassette component of the Escherichia coli maltodextrin transporter, inhibits the transcriptional activator MalT by antagonizing inducer binding. J. Biol. Chem. 279, 33123-33130.

11. Boos, W. \& Böhm, A. (2000). Learning new tricks from an old dog: MalT of the Escherichia coli maltose system is part of a complex regulatory network. Trends Genet. 16, 404-9.

12. Peist, R., Koch, A., Bolek, P., Sewitz, S., Kolbus, T. \& Boos, W. (1997). Characterization of the aes gene of Escherichia coli encoding an enzyme with esterase activity. J. Bacteriol. 179, 7679-7686. 
13. Reidl, J. \& Boos, W. (1991). The malX malY operon of Escherichia coli encodes a novel enzyme II of the phosphotransferase system recognizing glucose and maltose and an enzyme abolishing the endogenous induction of the maltose system. $J$. Bacteriol. 173, 4862-4876.

14. Vidal-Ingigliardi, D., Richet, E., Danot, O. \& Raibaud, O. (1993). A small C-terminal region of the Escherichia coli MalT protein contains the DNA-binding domain. $J$. Biol. Chem. 268, 24527-24530.

15. Danot, O. (2001). A complex signaling module governs the activity of MalT, the prototype of an emerging transactivator family. Proc. Natl. Acad. Sci. USA 98, 435440 .

16. Schlegel, A., Danot, O., Richet, E., Ferenci, T. \& Boos, W. (2002). The N-terminus of the Escherichia coli transcription activator MalT is the domain of interaction with MalY. J. Bacteriol. 184, 3069-3077.

17. Steegborn, C., Danot, O., Huber, R. \& Clausen, T. (2001). Crystal structure of transcription factor MalT domain III: A domain helix repeat fold implicated in regulated oligomerization. Structure 9, 1051-1060.

18. Larquet, E., Schreiber, V., Boisset, N. \& Richet, E. (2004). Oligomeric assemblies of the Escherichia coli MalT transcriptional activator revealed by cryo-electron microcopy and image processing. J. Mol. Biol. 343, 1159-1169.

19. Zhou, Y., Zhang, X. \& R.H., E. (1991). Random mutagenesis of gene-sized DNA molecules by use of PCR with Taq DNA polymerase. Nucleic Acids Res. 19, 6052.

20. Böhm, A., Diez, J., Diederichs, K., Welte, W. \& Boos, W. (2002). Structural model of $\mathrm{MalK}$, the ABC subunit of the maltose transporter of Escherichia coli: implications for mal gene regulation, inducer exclusion and subunit assembly. J. Biol. Chem. 277, 3708-3717.

21. Chen, J., Lu, G., Lin, J., Davidson, A. L. \& Quiocho, F. A. (2003). A tweezers-like motion of the ATP-binding cassette dimer in an ABC transport cycle. Mol. Cell 12, 651-61.

22. Locher, K. P., Lee, A. T. \& Rees, D. C. (2002). The E. coli BtuCD structure: a framework for ABC transporter architecture and mechanism. Science 296, 1091-8.

23. Panagiotidis, C. H., Boos, W. \& Shuman, H. A. (1998). The ATP-binding cassette subunit of the maltose transporter MalK antagonizes MalT, the activator of the Escherichia coli mal regulon. Mol. Microbiol. 30, 535-546.

24. Austermuhle, M. I., Hall, J. A., Klug, C. S. \& Davidson, A. L. (2004). Maltosebinding protein is open in the catalytic transition state for ATP hydrolysis during maltose transport. J. Biol. Chem. 279, 28243-50.

25. D'Andrea, L. D. \& Regan, L. (2003). TPR proteins: the versatile helix. Trends Biochem. Sci. 28, 655-62. 
26. Casadaban, M. J. (1976). Transposition and fusion of the lac genes to selected promoters in Escherichia coli using bacteriophage lambda and Mu. J. Mol. Biol. 104, 541-55.

27. Cole, S. T. \& Raibaud, O. (1986). The nucleotide sequence of the malT gene encoding the positive regulator of the Escherichia coli maltose regulon. Gene 42, 201-8.

28. Norregaard-Madsen, M., McFall, E. \& Valentin-Hansen, P. (1995). Organization and transcriptional regulation of the Escherichia coli K-12 D-serine tolerance locus. $J$. Bacteriol. 177, 6456-61.

29. Sambrook, J., Fritsch, E. F. \& Maniatis, T. (1989). Molecular cloning. 2nd edition edit, Cold Spring harbor Laboratory press, Cold Spring Harbor, N.Y.

30. Miller, J. H. (1972). Experiments in Molecular Genetics, Cold Spring Harbor Laboratory, Cold Spring Harbor, NY.

31. McClure, W. R. (1980). Rate-limiting steps in RNA chain initiation. Proc. Natl. Acad. Sci. USA 77, 5634-5638.

32. Hofnung, M., Hatfield, D. \& Schwartz, M. (1974). malB region of Escherichia coli K12: characterization of new mutations. J. Bacteriol. 117, 40-47.

33. Ehrmann, M. \& Boos, W. (1987). Identification of endogenous inducers of the mal regulon in Escherichia coli. J. Bacteriol. 169, 3539-3545. 


\section{LEGENDS TO THE FIGURES}

Figure 1. Location of the H10/H11 motif on the three-dimensional structure of DT3. The three-dimensional structure of DT3 is represented as a white molecular surface. Amino acid residues of the H10-H1 1motif are indicated by different colors. Inset: ribbon representation of helices H9 to H12, i.e. the fifth and sixth helix bundles. The same color code is used to highlight the side-chains of the residues of the H10/H11 motif.

Figure 2. In vivo activity of the up mutants in the absence of repressor or in the presence of MalK, MalY, or Aes. A, The amount of ß-galactosidase made by isogenic $\Delta$ malT $\Delta$ malK (no repressor), $\Delta$ malT malK ${ }^{+}$(+ MalK), or $\Delta$ malT $\Delta$ malK mall::Tn10 (+ MalY) strains (pop7190, pop7191 and pop7192, respectively), harboring derivatives of pOM181 was determined as described. The results are expressed relative to the unrepressed level obtained with wild-type MalT in pop7190 (3 333 U). B, The amount of B-galactosidase made by strain pop7190 harboring pOM181 derivatives, and pACYC184 (no Aes) or pACS2 (+ Aes) was determined as described. The results are expressed relative to the level obtained with wild type MalT in the absence of Aes (2 $515 \mathrm{U})$.

Figure 3. Effect of the MalT-N637T substitution on the inhibition of MalT activity by MalK, in vitro. Abortive initiation assays were performed as described in the presence of wild-type MalT (300 nM) (open squares) or MalT-N637T (300 nM) (closed squares), AMP-PNP $(0.5 \mathrm{mM})$ and the indicated concentration of MalK. All of the assays were performed in duplicate. Note that AMP-PNP can replace ATP as a positive effector of MalT ${ }^{2}$ and that the AMP-PNP bound form is partially active in the absence of maltotriose (N.J., unpublished results), hence the omission of the inducer. The background signal observed in the absence of MalT $(\leq 0.5$ ApApC/promoter/min) was substracted.

Figure 4. Effect of the MalT-N637T substitution on the inhibition of MalT activity by MalY and Aes, in vitro. Abortive initiation assays were performed as described in the presence of wild-type MalT (300 nM) (open squares) or MalT-N637T (300 nM) (closed squares) and the indicated concentration of MalY (A) or Aes (B).

Figure. 5. Effect of the MalT-N637T substitution on the MalT/MalK interaction in vitro. Purified MalT was chromatographed in the presence of ATP on Ni-NTA-agarose microcolumns preloaded with His-tagged MalK as described. The fractions recovered were analysed by SDS-PAGE and the proteins stained with Coomassie blue. FT, $W$, and $E$ are flow-through $(200 \mu \mathrm{l})$, wash fractions $(100 \mu \mathrm{l}$ each), and imidazole-eluted fractions $(100 \mu \mathrm{l}$ each), respectively. A, wild-type MalT. B, MalT-N637T. 
Click here to download high resolution image

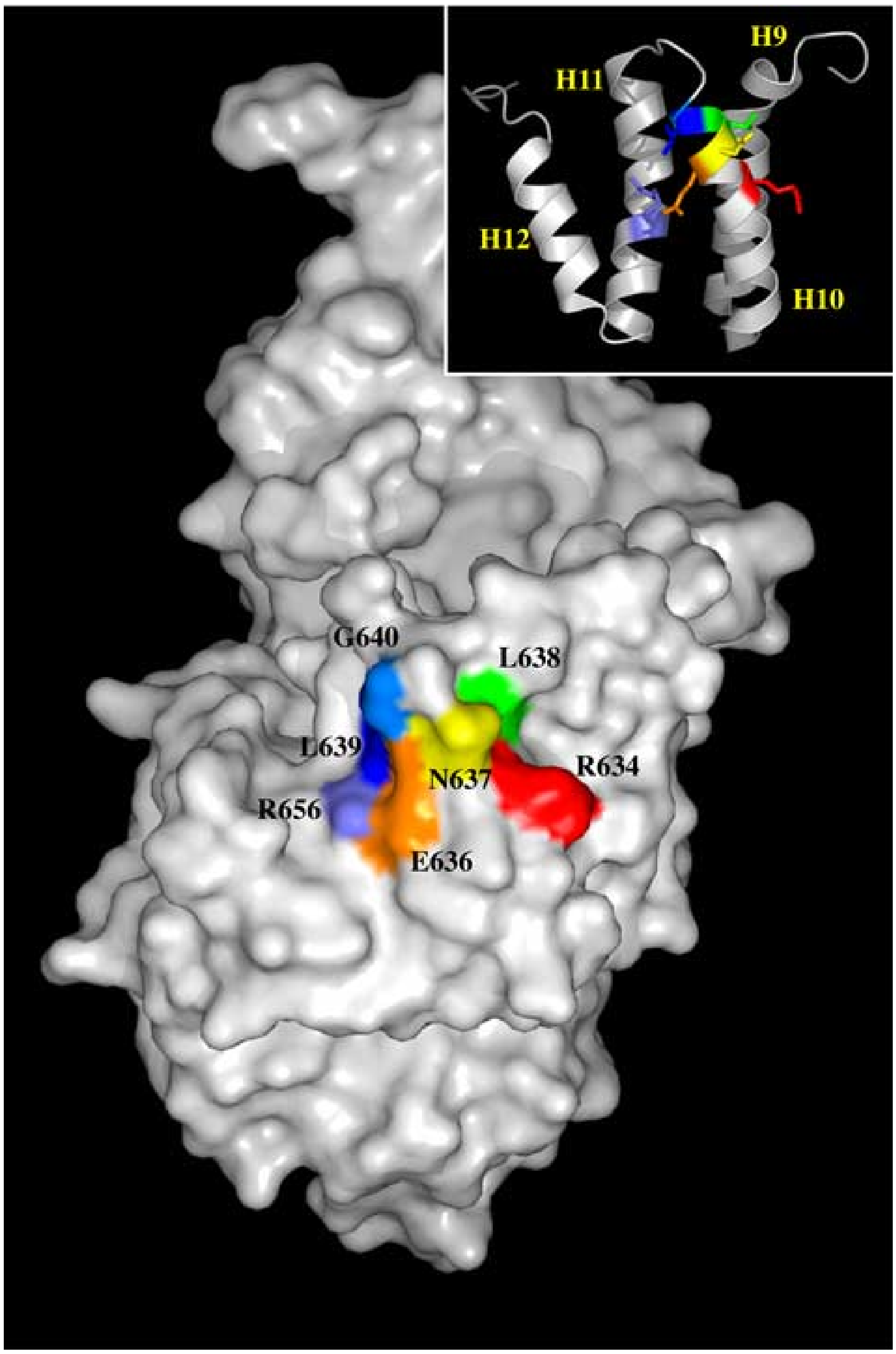


Fig. 2

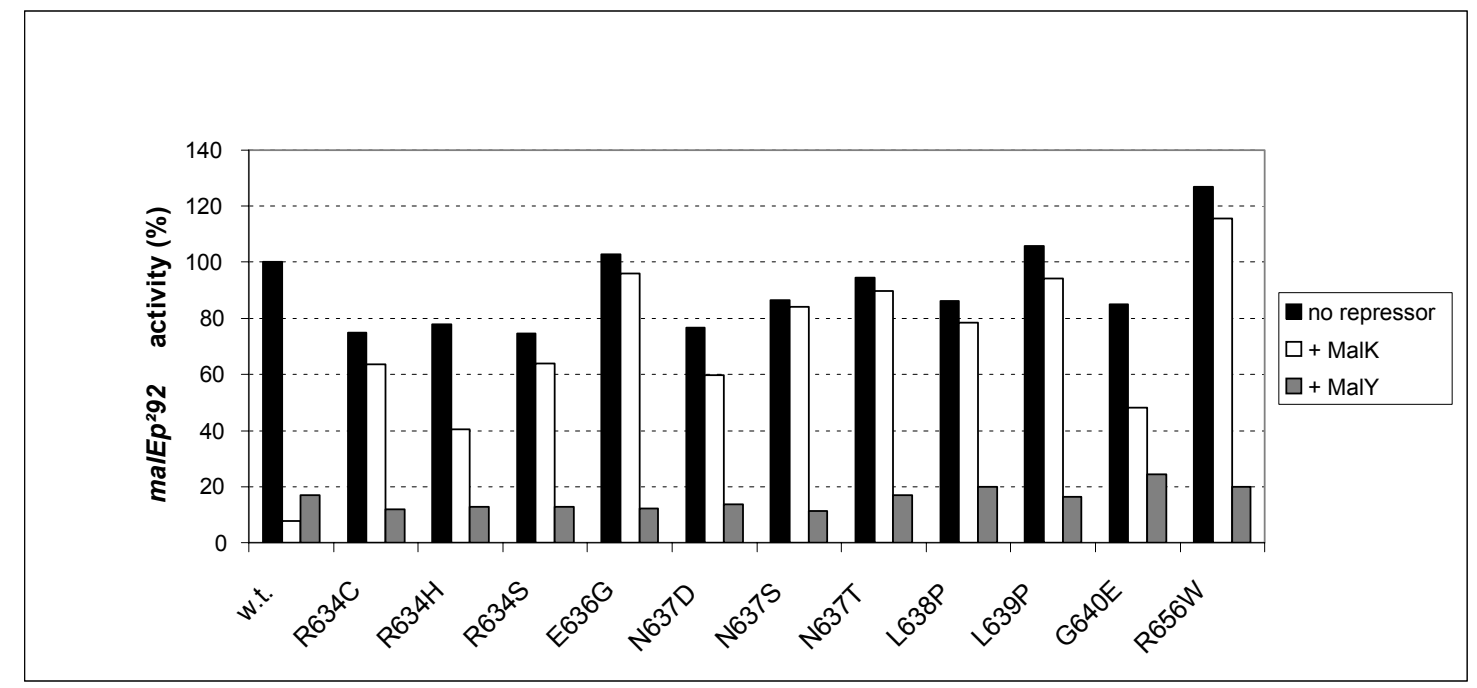

Fig. 2A

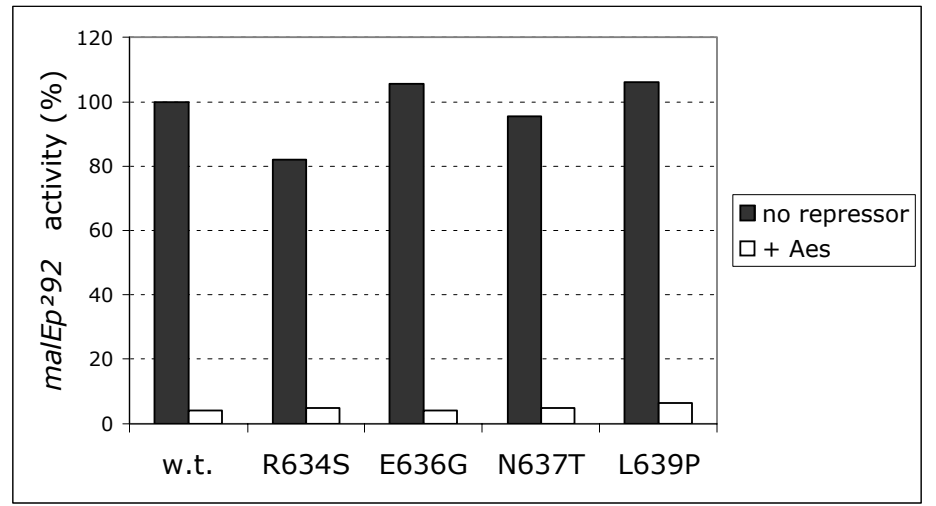

Fig. 2B 
Click here to download high resolution image

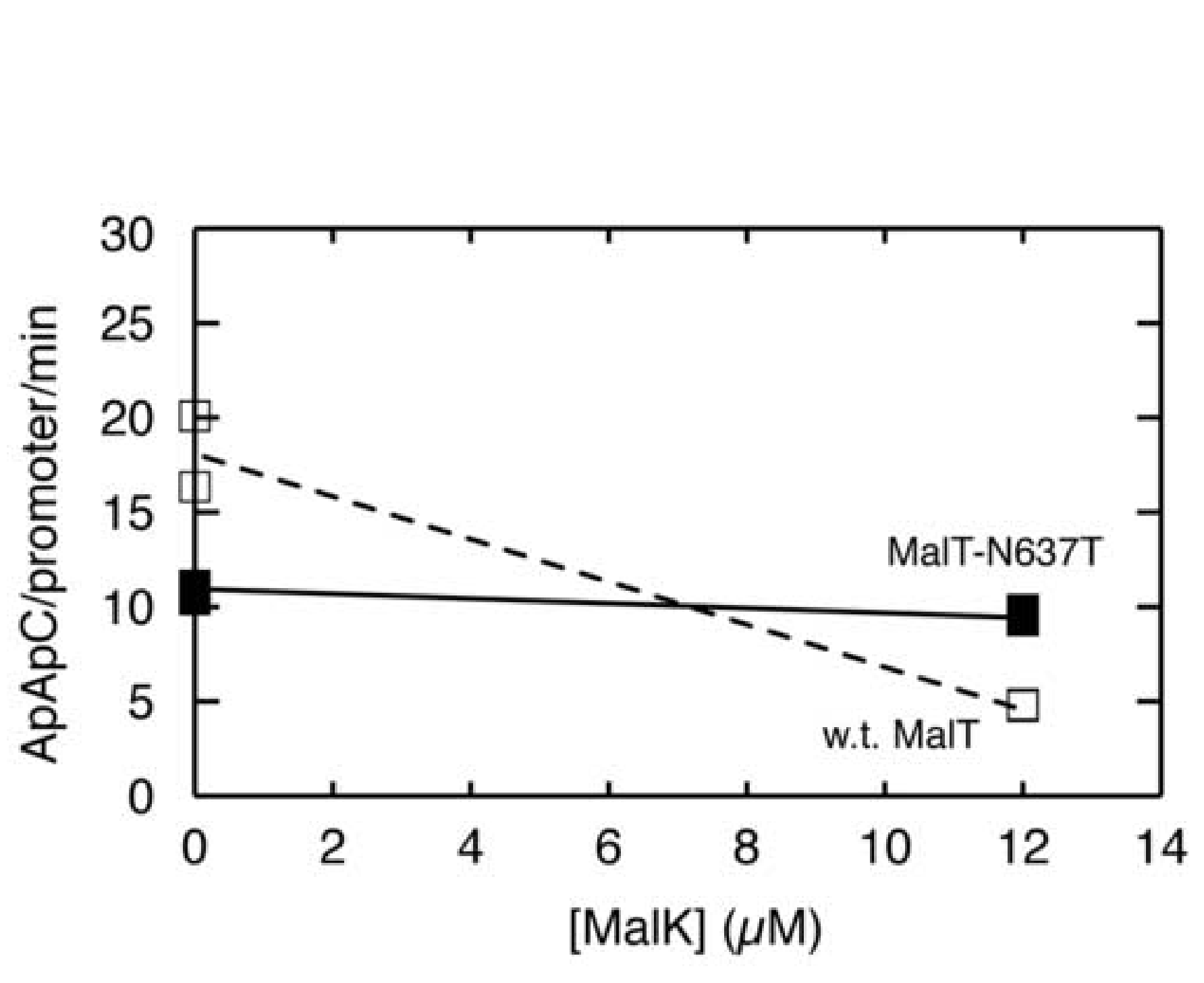

Fig. 3

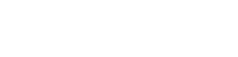

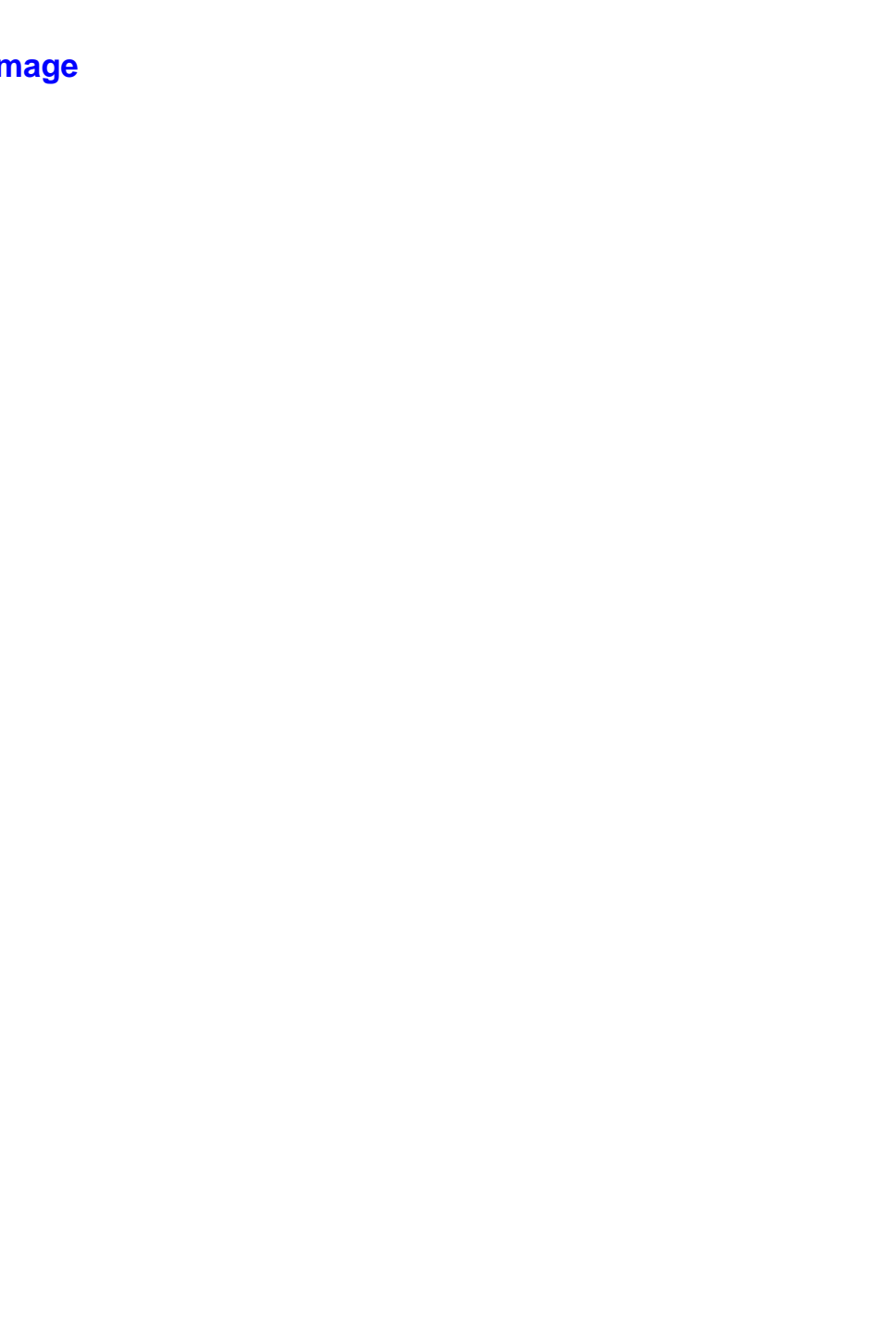


Fig. 4
Click here to download high resolution image
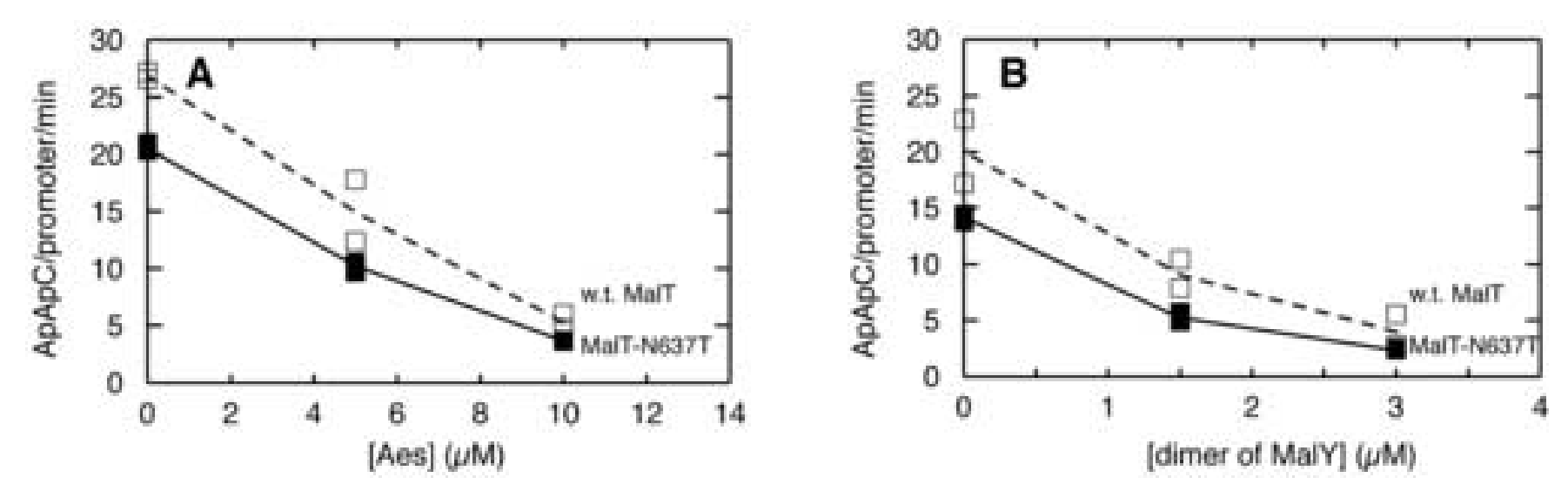

Fig. 4

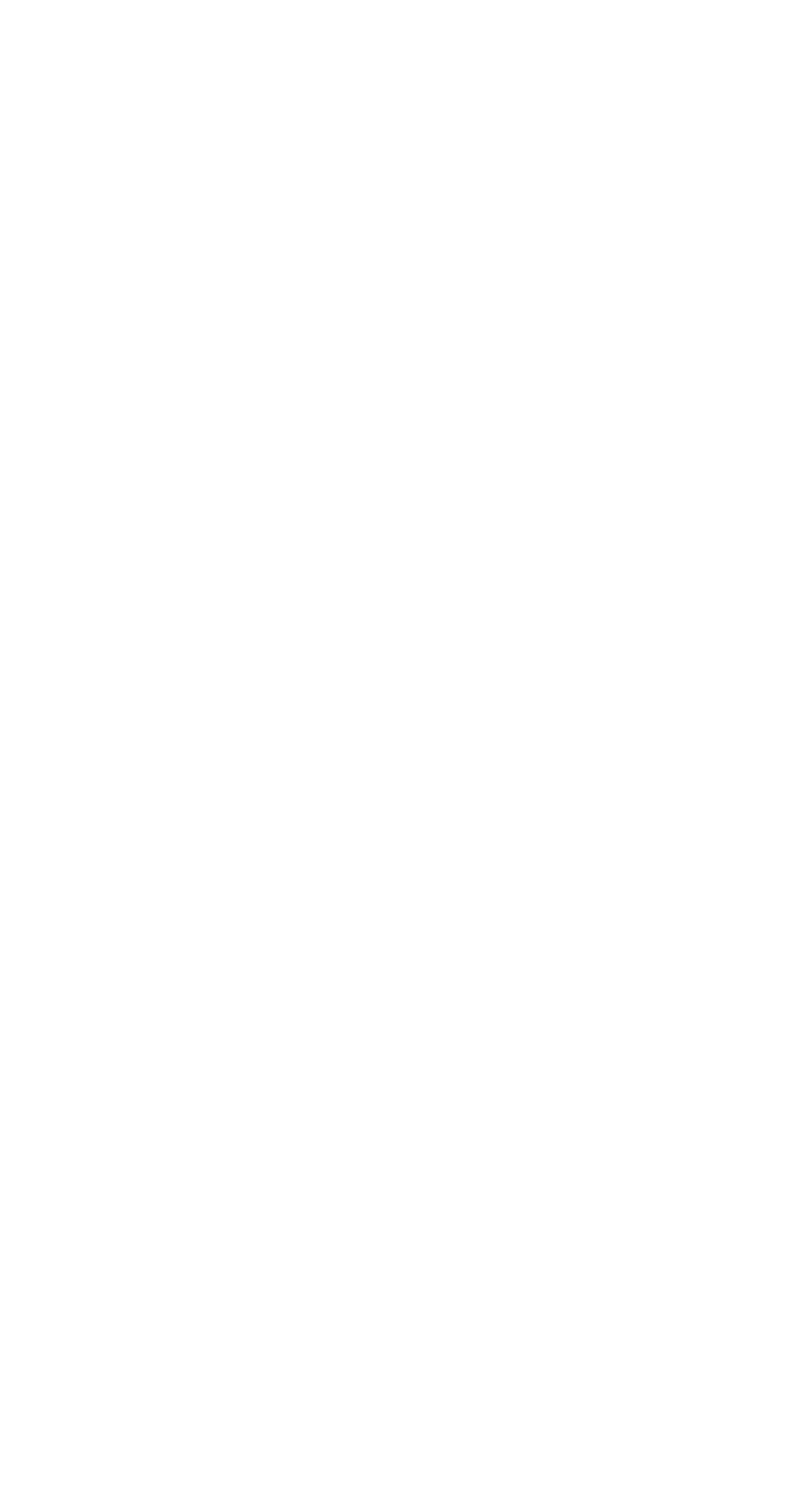

(a)

(c) of maly)

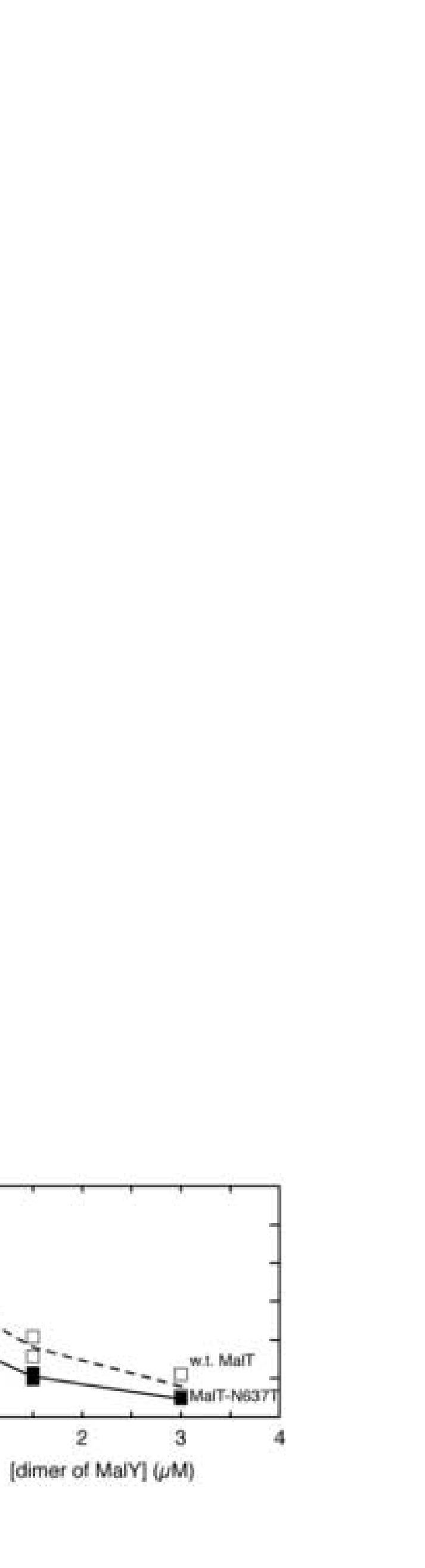


Fig. 5
Click h

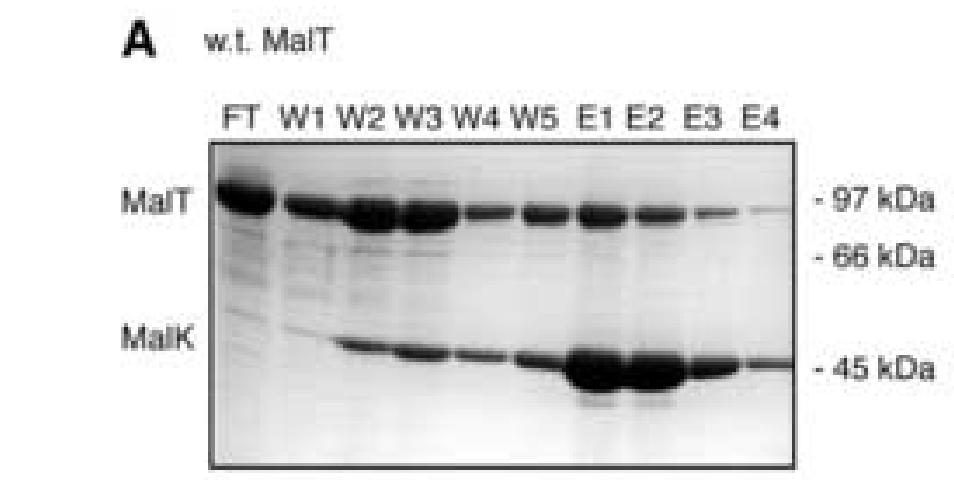

B MalT-N637T

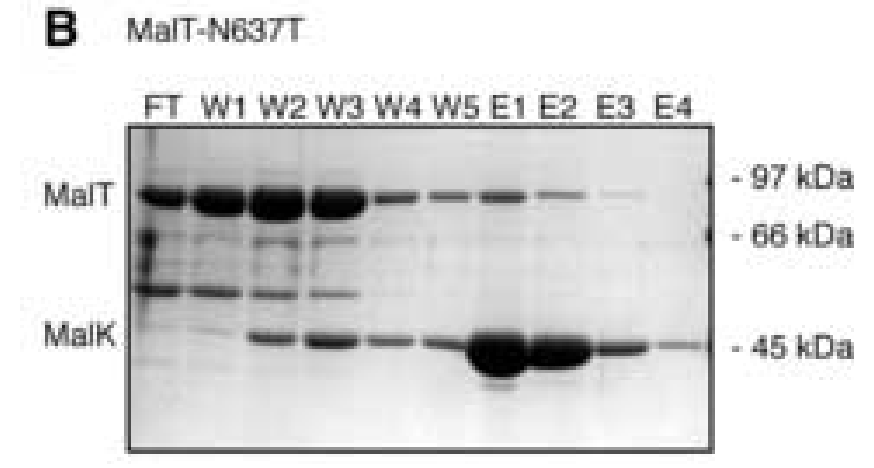

Click here to download high resolution image
A w.t. MalT
Fig. 5
Click

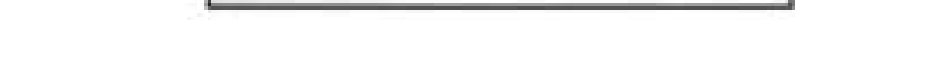

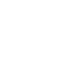

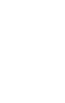


TABLE 1. Up mutants isolated

\begin{tabular}{|c|c|c|}
\hline Substitutions ${ }^{\mathrm{a}}$ & $\mathrm{N}^{\mathrm{b}}$ & Codon changes $^{c}$ \\
\hline M517K/H808R & 1 & ATG $->$ AAG $/$ CAT $->$ CGT \\
\hline Q565R & 1 & $\mathrm{CAG}->\mathrm{CGG}$ \\
\hline R634C & 1 & CGT -> TGT \\
\hline R634H & 1 & CGT $->$ CAT \\
\hline R634S & 1 & CGT $->$ AGT \\
\hline E636G & 2 & GAA -> GGA \\
\hline N637D & 3 & $\mathrm{AAC}->\mathrm{GAC}$ \\
\hline N637D/Q608R & 1 & $\mathrm{AAC}->\mathrm{GAC} / \mathrm{CAG}->\mathrm{CGG}$ \\
\hline N637S & 3 & $\mathrm{AAC}->\mathrm{AGC}$ \\
\hline N637T & 1 & $\mathrm{AAC}->\mathrm{ACC}$ \\
\hline L638P & 2 & $\mathrm{CTG}->\mathrm{CCG}$ \\
\hline L639P & 3 & $\mathrm{CTG}->\mathrm{CCG}$ \\
\hline L639P/E636E & 1 & CTG $->$ CCG / GAA $->$ GAG \\
\hline L639P/E705V & 1 & CTG -> CCG / GAA -> GTA \\
\hline G640E & 2 & GGG -> GAG \\
\hline G640R/Q792R & 1 & GGG $->$ AGG / CAG $->$ CGG \\
\hline R656W & 1 & CGG -> TGG \\
\hline R656W/H808R & 1 & CGG $->$ TGG / CAT $->$ CGT \\
\hline F810L & 1 & TTC -> CTC \\
\hline
\end{tabular}

${ }^{a}$ The residue number refers to its position in the MalT polypeptide.

${ }^{\mathrm{b}} \mathrm{N}$, number of independent isolates

${ }^{c}$ The percentage of transitions and transversions were 85 and $15 \%$, respectively. 
TABLE 2. Strains

\begin{tabular}{|c|c|c|}
\hline \multicolumn{2}{|c|}{ Strains Genotype } & \multirow{2}{*}{$\begin{array}{l}\text { References } \\
26\end{array}$} \\
\hline MC4100 & araD139 $\Delta(\operatorname{argF-lac}) U 169 \mathrm{rpsL} 150$ relA1 flbB5301 deoC1 ptsF25 rbsR & \\
\hline pop7164 & MC4100 $\Delta m a l T 220^{\mathrm{a}}$ & 8 \\
\hline ED169 & $\mathrm{MC} 4100 \Delta m a l B 107^{\mathrm{b}}$ & $\begin{array}{l}\text { from E. Dassa } \\
\text { (Institut Pasteur) }\end{array}$ \\
\hline pop7165 & MC4100 malB107 trp::[Kan ${ }^{\mathrm{r}}-$ malEp$\left.\Delta 92-l a c\right]_{\mathrm{op}}$ & This study ${ }^{c}$ \\
\hline pop7170 & MC4100 malB107 trp::[Kan $\left.{ }^{\mathrm{r}}-m a l E p \Delta 92-l a c\right]_{\mathrm{op}} \operatorname{aroB} \operatorname{glpD}$ & This study $^{d}$ \\
\hline pop7180 & MC4100 trp::[Kan $\left.{ }^{\mathrm{r}}-m a l E p \Delta 92-l a c\right]_{\mathrm{op}}$ aroB $g l p D$ & This study ${ }^{\mathrm{e}}$ \\
\hline pop7185 & MC4100 malB107 mall::Tn10 trp::[Kan ${ }^{\mathrm{r}}-$ malEp$\left.\Delta 92-l a c\right]_{\mathrm{op}}$ aroB glpD & This study ${ }^{\mathrm{f}}$ \\
\hline pop7190 & MC4100 malB107 trp::[Kan $\left.{ }^{\mathrm{r}}-m a l E p \Delta 92-l a c\right]_{\mathrm{op}} \Delta$ malT220 & This study ${ }^{\mathrm{g}}$ \\
\hline pop7191 & MC4100 trp::[Kan $\left.{ }^{\mathrm{r}}-m a l E p \Delta 92-l a c\right]_{\mathrm{op}} \Delta$ malT220 & This study ${ }^{\mathrm{h}}$ \\
\hline pop7192 & 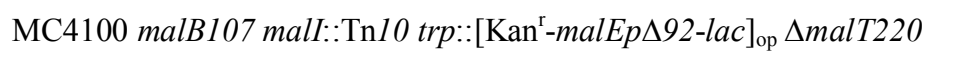 & This study ${ }^{\mathrm{i}}$ \\
\hline
\end{tabular}

${ }^{\text {a }} \triangle$ malT220 is a deletion of the entire malT gene ${ }^{8}$.

${ }^{\mathrm{b}} \Delta$ malB107 deletes most, if not all, of the malK gene ${ }^{32}$.

${ }^{\mathrm{c}}$ The locus trp::(Kan ${ }^{\mathrm{r}}-$ malEp$\left.\Delta 92-l a c\right)_{\text {op }}$ was engeneered and introduced into ED169 to give pop7165, as described ${ }^{16}$.

${ }^{d}$ Strain pop7170 was obtained by transduction of a malT::Tn10 derivative of pop 7165 to Aro$\mathrm{Tet}^{\mathrm{S}} \mathrm{Mal}^{+}$Gly” by using a P1 lysate of pop2322 (MC4100 aroB glpD) (Laboratory collection). ${ }^{\mathrm{e}}$ pop7180 is a $\mathrm{malB}^{+}$derivative of pop7170, constructed by $\mathrm{P} 1$ transduction from MC4100.

${ }^{\mathrm{f}}$ pop7185 is a mall::Tn10 ${ }^{33}$ derivative of pop7170, obtained by P1 transduction from MC4100 mall::Tn10 (Laboratory collection).

g Strain pop7190 is a $\Delta$ malT220 derivative of pop7170, constructed by P1 transduction to $\mathrm{Aro}^{+} \mathrm{Mal}^{-} \mathrm{Gly}^{+}$with a P1 lysate from pop7163 (MC4100 $\Delta$ malT220 $\left.\left(\mathrm{F}^{+}\right)\right)^{8}$.

${ }^{\mathrm{h}}$ Strain pop7191 is a $\Delta$ malT220 derivative of pop7180, obtained as described for pop 7190.

${ }^{\mathrm{i}}$ Strain pop7192 is a $\Delta$ malT220 derivative of pop7185, obtained as decribed for pop 7190. 